\title{
Comparative insecticidal efficacy of a new pyrethroid, metofluthrin, against colonies of Asian Culex quinquefasciatus and Culex pipiens pallens
}

\author{
Tamara Belzabel Obispo Argueta ${ }^{1)}$, Hitoshi KawAdA ${ }^{2)}$, \\ Masayo Sugano ${ }^{3)}$, Shun-ichi Kubota ${ }^{3)}$, Yoshinori Shono ${ }^{3)}$, \\ Kazunori TsushIma ${ }^{3)}$ and Masahiro TAKAGI ${ }^{2)}$ \\ 1) Ministry of Public Health and Social Assistance, Verbena, Guatemala \\ 2) Department of Vector Ecology \& Environment, Institute of Tropical Medicine, \\ Nagasaki University, Nagasaki, Nagasaki, 852-8523 Japan \\ 3) Agricultural Chemicals Research Laboratory, Sumitomo Chemical Co., Ltd., \\ Takarazuka, Hyogo, 665-8555 Japan
}

(Received: 30 April 2004; Accepted: 9 August 2004)

\begin{abstract}
Comparative insecticidal efficacy of metofluthrin, a newly synthesized pyrethroid, and other pyrethroids against several colonies of Asian Culex quinquefasciatus (from Indonesia, Thailand, Vietnam and Malaysia) was evaluated by topical application. Metofluthrin was the most effective against the four colonies of $C x$. quinquefasciatus. The $\mathrm{LD}_{50}$-based relative effective ratio of metofluthrin against $d$-allethrin was higher in Cx. quinquefasciatus (33.3 to 78.8) than in $C x$. pipiens pallens (27.8). The Vietnam colony was the most susceptible among the colonies in the study.
\end{abstract}

Key words: metofluthrin, transfluthrin, Culex quinquefasciatus, topical application, susceptibility

\section{INTRODUCTION}

Culex quinquefasciatus Say is an ubiquitous species and is abundant in tropical and subtropical countries. Cx. quinquefasciatus is highly anthropophilic, feeds readily both indoors and outdoors and has its peak biting period around midnight (Kettle, 1995). Lack of proper and adequate disposal of wastes, poor city planning and resource limitations appear to be the contributing factors encouraging propagation of this mosquito (Ali et al., 1999). It has been playing an important role as a biting nuisance in urban areas as well as its main role as a vector of bancroftian filariasis.

Metofluthrin (S-1264) is a newly synthesized pyrethroid, which has strong knockdown and lethal activities against mosquitoes (Shono et al., 2004; Sugano et al., 2004; Ujihara et al., 2004). The vapor pressure of metofluthrin is ca. $>2$ times and $>100$ times higher than that of $d$ allethrin and permethrin, respectively (Kawada et al., 2004a), making it vaporize at room temperature, while the other conventional pyrethroids need heating for evaporation. Kawada et al. (2004a, 2004b) reported the promising efficacy of new mosquito-controlling devices using metofluthrin (metofluthrin impregnated multilayer paper strip), which needs no external energy for vaporization, at low cost against $C x$. quinquefasciatus in Lombok Island, Indonesia. Transfluthrin also belongs to a pyrethroid group which has high volatility and high knockdown and lethal activities. Pates et al. (2002) reported unique attempts using a transfluthrinmixed kerosene oil lamp to repel the biting of $C x$. quinquefasciatus in the typical houses in Dar es Salaam, Tanzania. 
The two unique pyrethroids mentioned above will constitute a new era in the field of spatial repellent for insects, as well as in the field of conventional mosquitoproofing devices, such as mosquito coil, mat, and vaporized liquid. In this paper, we report on the comparative insecticidal activity of metofluthrin, transfluthrin and other pyrethroids against several colonies of Asian Cx. quinquefasciatus.

\section{Materials And Methods}

Three colonies of field-collected (from Indonesia, Thailand and Vietnam) and one laboratory colony of $C x$. quinquefasciatus and one laboratory colony of Culex pipiens pallens L. were used for the study. The Indonesia colony (IN) was collected at Mataram, Lombok Island, in February, 2003; the Thailand colony (TL) was collected at Mae Joh, Chiang Mai, in March, 2003; the Vietnam colony (VN) was collected at Catque, Hatay, in March, 2003. After collection, they were reared for several generations in the laboratory and the colonies of the fourth to sixth generations after collection were used for the study. The Malaysia colony (ML) of Cx. quinquefasciatus, introduced from School of Biological Sciences, Universiti Sains Malaysia (Foo and Yap, 1982), and the Gose colony of Cx. pipiens pallens, collected at Gose,
Nara, Japan (Mukai et al., 1974; Shinjo et al., 1981), were standard colonies which are susceptible to most insecticides. Mosquitoes were reared in the laboratory at $25^{\circ} \mathrm{C}, 70 \% \mathrm{RH}$ and $16 \mathrm{~L} 8 \mathrm{D}$ photoperiod regime.

Technical grades of metofluthrin, $(2,3$, 5,6 -tetrafluoro- 4 -methoxymethylbenzyl $(E, Z)(1 R, 3 R)$-2,2 -dimethyl-3-(prop-2-enyl) cyclopropanecarboxylate), transfluthrin, $d$-allethrin, permethrin, and fenitrothion were used for the study.

Female mosquitoes were anesthetized briefly with carbon dioxide and were put on the metal plate, the surface temperature of which was maintained $<4^{\circ} \mathrm{C}$ with ice, to keep the anesthesia. A $0.3 \mu \mathrm{g}$ acetone solution of the test chemical was topically applied to the dorsal mesothorax of 4- to 6-day-old female adults by Automatic Microapplicator (Burkard Manufacturing Co., Ltd., Hertfordshire, UK). The same amount of acetone without insecticide was treated to the females as untreated control. Ten insects treated were kept in a plastic cup, the bottom of which was lined with a filter paper, fed with 3\% sugar solution under the laboratory conditions noted above. Mortality was observed $24 \mathrm{~h}$ after treatment. Data obtained were corrected by the mortality of untreated insects with Abbott's formula and $\mathrm{LD}_{50}$ and $\mathrm{LD}_{90}$ was calculated by Bliss' Probit

Table 1. $\mathrm{LD}_{50} \mathrm{~S}$ of insecticides for several Asian strains of Culex quinquefasciatus and Culex pipiens pallens by topical application.

\begin{tabular}{cccccc}
\hline \hline \multirow{2}{*}{ Chemicals } & \multicolumn{4}{c}{$\mathrm{LD}_{50}(\mu \mathrm{g} / \mathrm{female})-(95 \%$ confidential limit $)$} \\
\cline { 2 - 5 } & \multicolumn{4}{c}{ Culex quinquefasciatus } & Culex pipiens pallens \\
\cline { 2 - 5 } & Indonesia & Thailand & Vietnam & Malaysia & Gose \\
\hline Metofluthrin & 0.0012 & 0.00080 & 0.00039 & 0.00033 & 0.0018 \\
& $(0.00086-0.0016)$ & $(0.00067-0.00095)$ & $(0.00026-0.00055)$ & $(0.00029-0.00037)$ & $(0.0013-0.0026)$ \\
Transfluthrin & 0.0053 & 0.0033 & 0.0024 & 0.0024 & 0.0066 \\
d-Allethrin & $(0.0033-0.0072)$ & $(0.0027-0.0043)$ & $(0.0020-0.0028)$ & $(0.0018-0.0042)$ & $(0.0053-0.0082)$ \\
Permethrin & 0.057 & 0.063 & 0.013 & 0.013 & 0.050 \\
Fenitrothion & $(0.044-0.074)$ & $(0.049-0.088)$ & $(0.010-0.016)$ & $(0.0091-0.016)$ & $(0.040-0.059)$ \\
& 0.0018 & 0.011 & 0.0016 & 0.0044 & 0.0028 \\
& $(0.0066-0.0099)$ & $(0.0068-0.010)$ & $(0.0022-0.0042)$ & $(0.00071-0.0019)$ & $(0.0040-0.0065)$ \\
\hline
\end{tabular}


Table 2. $\mathrm{LD}_{90} \mathrm{~S}$ of insecticides for several Asian strains of Culex quinquefasciatus and Culex pipiens pallens by topical application.

\begin{tabular}{cccccc}
\hline \hline & \multicolumn{4}{c}{$\mathrm{LD}_{90}(\mu \mathrm{g} / \mathrm{female})-(95 \%$ confidential limit $)$} \\
\cline { 2 - 5 } Chemicals & \multicolumn{4}{c}{ Culex quinquefasciatus } & Culex pipiens pallens \\
\cline { 2 - 5 } & Indonesia & Thailand & Vietnam & Malaysia & Gose \\
\cline { 2 - 5 } Metofluthrin & 0.0050 & 0.0017 & 0.0014 & 0.00063 & 0.0049 \\
& $(0.0035-0.0091)$ & $(0.0014-0.0025)$ & $(0.00096-0.0027)$ & $(0.00054-0.00078)$ & $(0.0031-0.018)$ \\
Transfluthrin & 0.019 & 0.011 & 0.0053 & 0.0067 & 0.013 \\
d-Allethrin & $(0.012-0.086)$ & $(0.0074-0.020)$ & $(0.0044-0.0073)$ & $(0.0039-0.034)$ & $(0.010-0.022)$ \\
Permethrin & 0.16 & 0.30 & 0.044 & 0.034 & 0.088 \\
Fenitrothion & $(0.11-0.38)$ & $(0.18-0.84)$ & $(0.033-0.068)$ & $(0.025-0.061)$ & $(0.071-0.16)$ \\
& 0.0054 & 0.030 & 0.0050 & 0.015 & 0.0057 \\
& $(0.015-0.031)$ & $(0.020-0.045)$ & $(0.0091-0.020)$ & $(0.0029-0.023)$ & $(0.0090-0.022)$ \\
\hline
\end{tabular}

method (Bliss, 1934). Two to 6 replicates were made for each dosage.

\section{Results AND Discussion}

$\mathrm{LD}_{50} \mathrm{~S}$ and $\mathrm{LD}_{90} \mathrm{~s}$ of insecticides for several colonies of Asian Cx. quinquefasciatus and $C x$. pipiens pallens by topical application are shown in Tables 1 and 2. $\mathrm{LD}_{50} \mathrm{~S}$ of $d$-allethrin, permethrin and fenitrothion for Cx. pipiens pallens were 0.050, 0.0028 and $0.0054 \mu \mathrm{g} /$ female, respectively. They correspond well to the data in the prior reports for the same colony, in which $\mathrm{LD}_{50} \mathrm{~S}$ of $d$-allethrin, permethrin and fenitrothion were 0.037 (Shinjo et al., 1981), 0.0031 (Shinjo et al., 1989), and 0.0052 $\mu \mathrm{g} /$ female (Kawada, 1999), respectively. Dose-mortality regression lines for $C x$. quinquefasciatus and $C x$. pipiens pallens with metofluthrin, transfluthrin, and $d$-allethrin are shown in Fig. 1. Susceptibility of the standard colony of Cx. quinquefasciatus (ML) was slightly higher than that of the standard colony of Cx. pipiens pallens; $\mathrm{LD}_{50} \mathrm{~S}$ were ca 5.5, 2.8, and 3.8 times lower in $\mathrm{ML}$ colony than $C x$. pipiens pallens for metofluthrin, transfluthrin, and $d$-allethrin, respectively. Susceptibility of the VN colony seemed to be almost the same as that of the ML colony, indicating that the VN colony has experienced little exposure to pyrethroids and organophos- phates, or that the genes conferring resistance were absent in the VN colony. Susceptibility of the IN and TL colonies, on the other hand, was slightly lower than that of the ML and VN colonies; $L_{50}$ s for the IN and TL colonies were ca. 3.6 and 2.7 times with metofluthrin, 2.2 and 1.4 times with transfluthrin, 4.4 and 4.8 times with $d$-allethrin, higher than ML colony, respectively. Insecticide resistance, however, should not simply be attributed to the above differences in susceptibility, since there is little information on the susceptibility of $C x$. quinquefasciatus by topical application. Those differences in susceptibility might be deemed as due to variation among the collection sites. Metofluthrin was the most effective for all colonies among the insecticides tested in the study. Table 3 shows $\mathrm{LD}_{50}$-based relative effective ratio of metofluthin and transfluthrin against $d$-allethrin. The relative effective ratio for metofluthrin was higher in $C x$. quinquefasciatus (33.3 to 78.8) than in $C x$. pipiens pallens (27.8). On the contrary, differences in the relative effective ratio of transfluthrin against $d$-allethrin between $C x$. quinquefasciatus (5.4 to 19.1) and $C x$. pipiens pallens (7.6) seemed to be lower than those in metofluthrin.

Few studies on the insecticide susceptibility by topical application have been made for mosquitoes, since the method 

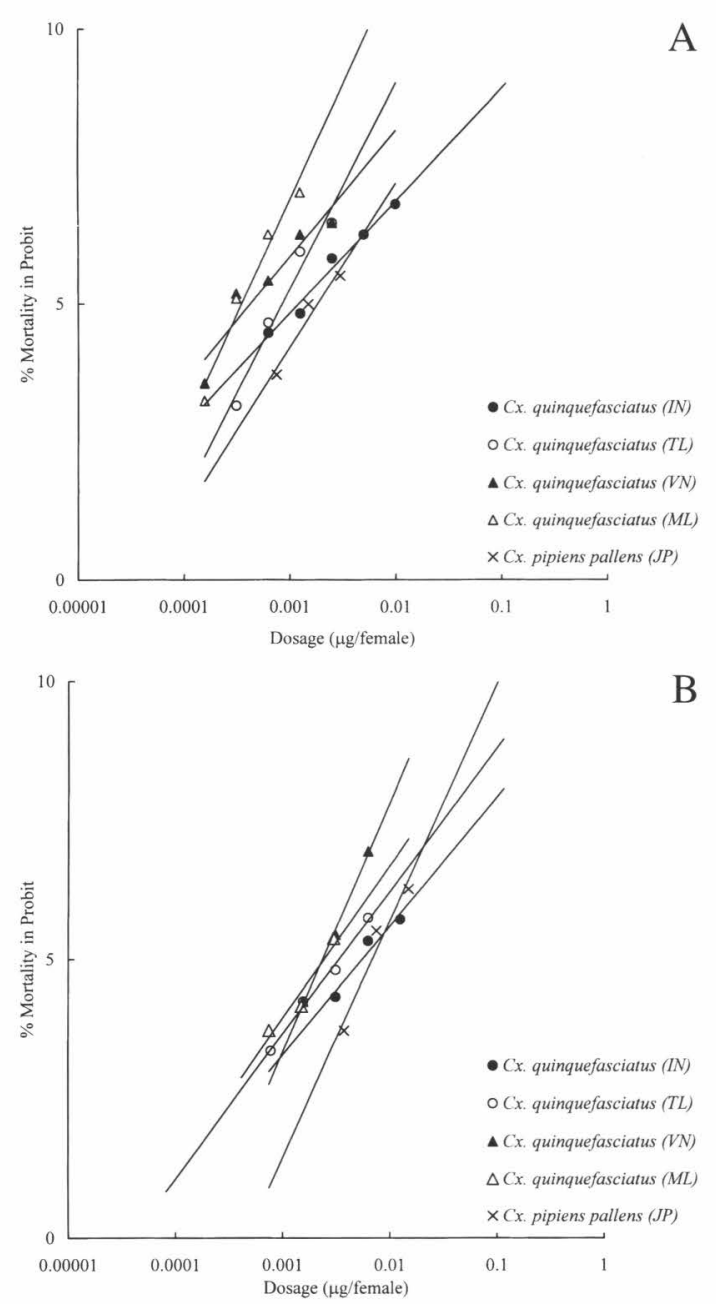

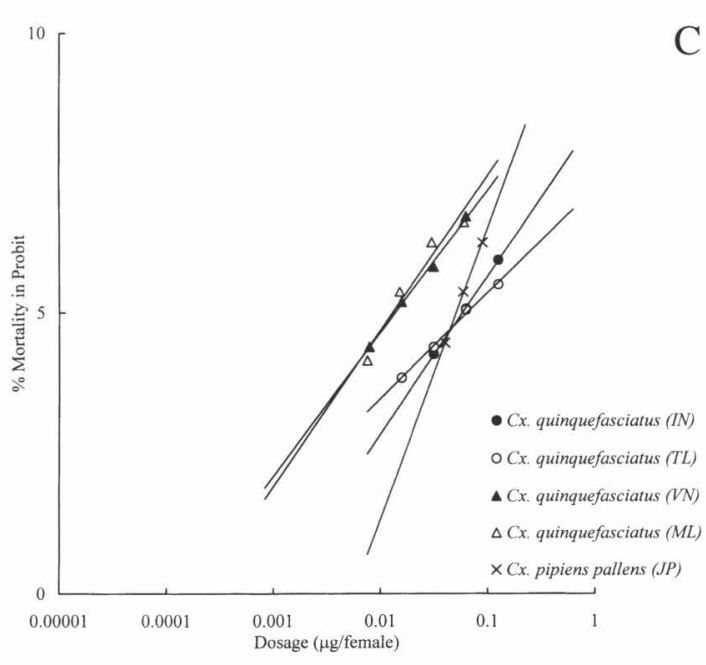

Fig. 1. Dose-mortality regression lines for Culex quinquefasciatus (IN, Indonesia colony; TL, Thailand colony; VN, Vietnam colony; ML, Malaysia colony) and $C x$. pipiens pallens (JP, Gose colony) with (A) metofluthrin, (B) transfluthrin, and (C) $d$-allethrin by topical application.

Table 3. $\mathrm{LD}_{50}$ based relative effective ratio of pyrethroids for several Asian strains of Culex quinquefasciatus and Culex pipiens pallens.

\begin{tabular}{|c|c|c|c|c|c|}
\hline \multirow{3}{*}{ Chemicals } & \multicolumn{5}{|c|}{ Relative effective ratio $(d$-Allethrin $=1.0)$} \\
\hline & \multicolumn{4}{|c|}{ Culex quinquefasciatus } & \multirow{2}{*}{$\frac{\text { Culex pipiens pallens }}{\text { Gose }}$} \\
\hline & Indonesia & Thailand & Vietnam & Malaysia & \\
\hline Metofluthrin & 47.5 & 78.8 & 33.3 & 39.4 & 27.8 \\
\hline Transfluthrin & 10.8 & 19.1 & 5.4 & 5.4 & 7.6 \\
\hline d-Allethrin & 1.0 & 1.0 & 1.0 & 1.0 & 1.0 \\
\hline
\end{tabular}

requires a well-trained technique and expensive apparatus which will be the most serious obstacles to carrying out such study in rural areas of tropical countries. More convenient and inexpensive methods, such as WHO test kits (Bruce-Chwatt, 1985), are recommendable in such areas. Topical application, however, seems to be the best way to get preliminary information, such as the transition in development of insecticide resistance or the relative efficacy ratio of insecticides (Suzuki, 1963; Kawada et al., 1993). The present results indicate that metofluthrin will be one of the most promising chemicals for controlling mosquitoes with its unique character- 
istics and high activity. Kawada et al. (2004a) reported that a single multilayer strip of metofluthrin-impregnated paper caused high knockdown to the caged Anopheles balabacensis Baisas, and all insects knocked down in less than $30 \mathrm{~min}$ in the indoor conditions. The authors also observed that mosquitoes were affected by airborne metofluthrin vapor and not by direct contact to the chemical, resulting in the spatial repellency (Argueta et al., 2004; Kawada et al., 2004a, 2004b). The spatial repellency is thought to take place with two main modes of pyrethroid action, i.e., knockdown activity and biting inhibition or disruption of orientation to the host. Among them, the latter may be categorized as a sublethal effect that resulted in neural excitement, which probably took place in the earlier stage of treatment or with lower dosage than that required for knockdown or death (MacIver, 1964; Winney, 1975; Birley et al., 1987). Topical application, which is the best way for the evaluation of killing activity, may also be applicable to some extent for the evaluation of knockdown activity of pyrethroids. Topical application, however, seems not to be an adequate way for evaluation of such spatial repellency with the volatile chemicals, since such activity requires a balance of overall factors of chemicals, such as physico-chemical properties as well as biological activity. Another new method, therefore, will be required for further studies on the spatial repellency of pyrethroids.

\section{ACKNOWLEDGEMENTS}

We express our deep gratitude to Dr. T. Satoh, Faculty of Pharmaceutical Sciences, Fukuoka University, Ms. M. Hasegawa, Ms. E. Kawashima, and Ms. K. Shimabukuro, Institute of Tropical Medicine, Nagasaki University, Ms. A. Yamashita and Ms. Y. Inoue, Agricultural Chemicals Research Laboratory, Sumitomo Chemical Co., Ltd., Ms. Ng. T. Yen, National Institute of Hygiene and Epidemiology,
Vietnam for supplying the mosquito colonies and their assistance in the study. The first author is also grateful to Japan International Cooperation Agency for providing the opportunity to conduct this study and for the financial support in Japan.

\section{REFERENCES}

Ali, A., Chowdhury, M. A., Hossain, M. I., Mahmud-UlAmeen, Habiba, D. B. and Aslam, A. F. 1999. Laboratory evaluation of selected larvicides and insect growth regulators against field-collected Culex quinquefasciatus larvae from urban Dhaka, Bangladesh. J. Am. Mosq. Control Assoc., 15: 43-47.

Argueta, T. B. O., Kawada, H. and Takagi, M. 2004. Spatial repellency of metofluthrin-impregnated multilayer paper strip against Aedes albopictus in the outdoor conditions. Med. Entomol. Zool., 55: 211-216.

Birley, M. H., Mutero, C. M., Turner, I. F. and Chadwick, P. R. 1987. The effectiveness of mosquito coils containing esbiothrin under laboratory and field conditions. Ann. Trop. Med. Parasitol., 81: 163171.

Bliss, C. I. 1934. The method of probits. Science, 79: 38-39.

Bruce-Chwatt, L. J. 1985. Essential Malariology 2nd ed. 452 pp. John Wiley \& Sons, New York.

Foo, A. E. and Yap, H. H. 1982. Comparative bioassays of Bacillus thuringiensis $\mathrm{H}-14$ formulations against four species of mosquitoes in Malaysia. Southeast Asian J. Trop. Med. Public Health, 13: 206-210.

Kawada, H. 1999. Type and characteristics of insecticides. In: Health Supervision of Rats and Insect Pests. (ed. Mogi, Y.) pp. 222-237. Fuji TechnoSystem, Tokyo. (In Japanese).

Kawada, H., Maekawa, Y., Tsuda, Y. and Takagi, M. 2004a. Laboratory and field evaluation of spatial repellency with metofluthrin impregnated paper strip against mosquitoes in Lombok Island, Indonesia. J. Am. Mosq. Control Assoc, 20: 292-298.

Kawada, H., Maekawa, Y., Tsuda, Y. and Takagi, M. 2004b. Trial of spatial repellency of metofluthrin impregnated paper strip against Anopheles and Culex in shelters without walls in Lombok, Indonesia. J. Am. Mosq. Control Assoc, (in press).

Kawada, H., Shono, Y., Itoh, T. and Abe, Y. 1993. 
Laboratory evaluation of insect growth regulators against several species of anopheline mosquitoes. Jpn. J. Sanit. Zool., 44: 349-353.

Kettle, D. S. 1995. Medical and Veterinary Entomology 2nd ed. CAB International, Wallingford, Oxon.

MacIver, D. R. 1964. Mosquito coils Part II. Studies on the action of mosquito coil smoke on mosquitoes. Pyrethrum Post, 7: 7-14.

Mukai, S., Sogo, K. and Hayashi, A. 1974. The resistant level of the larvae of pale house mosquito, Culex pipiens pallens Coquillett to several synthetic insecticides in Amagasaki City. Botyu-Kagaku., 39: 91-93 (In Japanese).

Pates, H. V., Line, J. D., Keto, A. J. and Miller, J. E. 2002. Personal protection against mosquitoes in Dar es Salaam, Tanzania, by using a kerosene oil lamp to vaporize transfluthrin. Med. Vet. Entomol., 16: 277-284.

Shinjo, G., Yamaguchi, T., Tsuda, S., Yoshida, K. and Okuno, Y. 1981. A study on the insecticidal activity of $d$-tetramethrin. Jpn. J. Sanit. Zool., 32: 221228.

Shinjo, G., Yano, T., Matsuo, N., Umemura, T., Mitsuda, S. and Seki, T. 1989. Study on a new synthetic pyrethroid "Etoc ${ }^{\mathbb{1} "}$. Sumitomokagaku, 1989-II: 418 (In Japanese).
Shono, Y., Kubota, S., Sugano, M., Yap, H. H. and Tsushima, K. 2004. Field evaluation of papers strip and mosquito coil formulation impregnated metofluthrin for mosquito control in Malaysia. In: The Abstract Book, 70th Annual Meeting of the American Mosquito Control Association, p. 40. American Mosquito Control Association, Eatontown.

Sugano, M., Shono, Y., Ishiwatari, T. and Tsushima, K. 2004. Metofluthrin, new synthetic pyrethroids, an innovative mosquito control agent. In: The Abstract Book, 70th Annual Meeting of the American Mosquito Control Association, p. 6. American Mosquito Control Association, Eatontown.

Suzuki, T. 1963. Insecticide resistance in flies, mosquitoes and cockroaches in Japan evaluated by topical application tests, with special reference to the susceptibility levels of the insects. Jpn. J. Exp. Med., 33: 69-83.

Ujihara, K., Mori, T., Iwasaki, T., Sugano, M., Shono, Y. and Matsuo, N. 2004. Metofluthrin: A potent new synthetic pyrethroid with high vapor activity against mosquitoes:Biosci. Biotechnol. Biochem., 68: 170-174.

Winney, R. 1975. Pyrethrins and pyrethroids in coils -a review. Pyrethrum Post, 13: 17-22. 